\title{
Characterising the dynamics of expirated bloodstain pattern formation using high-speed digital video imaging
}

\author{
Andrea E. Donaldson • Nicole K. Walker • \\ Iain L. Lamont • Stephen J. Cordiner • \\ Michael C. Taylor
}

Received: 28 April 2010 / Accepted: 15 July 2010

(C) Springer-Verlag 2010

\begin{abstract}
During forensic investigations, it is often important to be able to distinguish between impact spatter patterns (blood from gunshots, explosives, blunt force trauma and/or machinery accidents) and bloodstain patterns generated by expiration (blood from the mouth, nose or lungs). These patterns can be difficult to distinguish on the basis of the size of the bloodstains. In this study, high-speed digital video imaging has been used to investigate the formation of expirated bloodstain patterns generated by breathing, spitting and coughing mechanisms. Bloodstain patterns from all three expiration mechanisms were dominated by the presence of stains less than $0.5 \mathrm{~mm}$ in diameter. Video analysis showed that in the process of coughing blood, high-velocity, very small blood droplets were ejected first. These were followed by lower velocity, larger droplets, strands and plumes of liquid held together in part by saliva. The video images showed the formation of bubble rings and beaded stains, traditional markers for classifying expirated patterns. However, the expulsion mechanism, the distance travelled by the blood droplets,
\end{abstract}

\footnotetext{
A. E. Donaldson $(\bowtie) \cdot$ I. L. Lamont

Department of Biochemistry, University of Otago,

P.O. Box 56, Dunedin, New Zealand

e-mail: donan998@student.otago.ac.nz

N. K. Walker

Department of Chemistry, University of Auckland,

Private Bag 92019, Auckland, New Zealand

S. J. Cordiner

Institute of Environmental Science and Research (ESR) Ltd, P.O. Box 50-348, Porirua, New Zealand

M. C. Taylor

Institute of Environmental Science and Research (ESR) Ltd, P.O. Box 29-181, Christchurch, New Zealand
}

and the type of surface the blood was deposited on were all factors determining whether beaded stains were generated.

Keywords Blood pattern analysis .

Expirated blood spatter - Beaded stains $\cdot$ Bubble rings .

High-speed digital imaging

\section{Introduction}

Expirated blood is blood forced by airflow out of the nose, mouth, or a wound [1]. Expirated bloodstain patterns usually occur when an individual with blood in the mouth or nose breathes or coughs expelling blood onto nearby surfaces. These patterns can sometimes be difficult to distinguish from impact spatter which results from an object striking liquid blood [1]. Small bloodstains on an individual's clothing could be the result of that person delivering blows to a victim. Alternatively, they could indicate that this individual was in fact rendering aid to the injured party and the bloodstains are the result of expiration, a finding which would tend to exonerate that individual. Distinguishing these mechanisms can therefore be very important. This distinction is most problematic with partial patterns of only a few bloodstains on absorbent surfaces such as clothing [2-5].

Expirated blood can sometimes be distinguished from impact spatter by identifying physical characteristics such as bubble rings [1], the result of blood being mixed with air as it is being expelled, 'beading' which is thought to be small blood drops being linked by strings of mucus or saliva and dilution by other body fluids, such as saliva [3, 6-8]. Stains can also be tested for the presence of saliva [9]. However, the absence of these characteristics does not necessarily indicate that the pattern is not from expirated 
blood [7, 8]. Recently high-speed video images have been produced showing the formation of a wide range of bloodstain patterns, including expirated patterns [10]. These images of expirated blood show a person in a standing position gently breath-expelling blood from the mouth and coughing blood from inside the mouth.

In this study, we explore the mechanism of the expiration of blood using a high-speed digital video camera. Images were captured of mouth expiration using coughing, spitting and breathing mechanisms. The appearance of the resulting bloodstain patterns as well as the distance that expirated blood droplets travelled, has been considered.

\section{Methods and materials}

Expirated bloodstains were produced by a volunteer participant transferring between 1 and $5 \mathrm{~mL}$ of blood into the mouth and then expelling it towards a target surface. Three mouth-expiratory mechanisms; coughing, spitting and breathing, were used. Blood was collected in 5-mL $\mathrm{K}_{2}$ EDTA (potassium ethylene diamine tetraacetic acid) treated vacutainer blood tubes (Becton-Dickinson), warmed to $37^{\circ} \mathrm{C}$ prior to use and used within $48 \mathrm{~h}$ of collection.

In the coughing experiments, blood was placed into the back of the mouth until the gag reflex was almost triggered. The head was held as still as possible in a neutral position before the blood was then coughed. Fourteen experiments were performed using this method. Three involved a participant lying prone on the floor and the remainder involved the participant standing at distances of $300 \mathrm{~mm}$ to $1 \mathrm{~m}$ from a cardboard target. Two of the prone experiments involved a second participant who was kneeling approximately $300 \mathrm{~mm}$ away from the first in a position designed to replicate an individual seeking to render aid to a victim. The target surfaces used in the coughing experiments were white cardboard, white cotton lab coat fabric and white fabric Tyvex ${ }^{\circledR}$ (DuPont ${ }^{\mathrm{TM}}$, China) overalls.

Three experiments were performed using a spitting mechanism. In the spitting experiments blood was placed into the middle of the mouth. The head was again held in neutral position before blood was spat out through pursed lips. Two experiments involved the participant being prone and one involved the participant standing. In the standing experiment the participant was approximately $150 \mathrm{~mm}$ from the cardboard target. The prone experiments involved a second participant $300 \mathrm{~mm}$ from the first in a kneeling position.

Two experiments were undertaken using gentle breathing as an expiration mechanism. For both experiments the participant was standing. In the first experiment, the participant dripped approximately $1 \mathrm{~mL}$ of blood externally down the nose and upper lip before breathing normally with a very slightly opened mouth. In the second experiment, the participant put $1 \mathrm{~mL}$ of blood into the mouth and gently breathed out the blood onto a white cardboard target placed approximately $150 \mathrm{~mm}$ away.

Digital video images of each experiment were collected using a Photron SA1 high-speed digital video camera. These images, along with the various target surfaces, were then examined. In each experiment the expirated droplets were observed in flight, and the size, shape, and distribution of the bloodstains in the resulting pattern were analysed. An area of $100 \mathrm{~mm} \times 100 \mathrm{~mm}$ on the target surface was selected to determine the average size distribution of the bloodstains. Calculation of the size of an individual bloodstain was done by placing a transparent metric ruler over the stain and measuring its diameter.

Lighting for the high-speed imaging was achieved with two $2,000-\mathrm{W}$ and one $1,000-\mathrm{W}$ quartz halogen lamps. A $55-\mathrm{mm}$ Nikkor lens was used for experiments involving the standing participant. When the participant was prone, a 28-mm Nikkor lens was used to give a wider field of view. Apertures between $\mathrm{f} 4$ and $\mathrm{f} 11$ were used depending on the available light at the shutter speed chosen to minimise motion blur $(50-100 \mu \mathrm{sec})$.

\section{Results}

\section{Coughing experiments}

In each of the coughed-expirated blood experiments, blood droplets were observed to leave the mouth in a particular sequence. Tiny mist-like droplets (generally $<1 \mathrm{~mm}$ in diameter) were expelled first followed by larger droplets (generally $>1 \mathrm{~mm}$ in diameter) and strands of liquid, travelling more slowly. The tiny mist-like droplets were projected out of the mouth in a cone shape, and in an upward and forward direction. The slower strands and droplets were projected forward out of the middle and top of the mouth. Some of these impacted on the front teeth causing tumbling droplets which continued to spin and rotate in flight. The strands appeared to form 'plumes' (very long, thick, strands) after they had made contact with the front teeth and lips (Fig. 1).

The formation of these 'plumes' is probably due to the presence of salivary mucin proteins. Salivary mucin proteins are known to contribute greatly to the viscoelastic nature of saliva as well as provide lubrication, prevent mechanical damage, protect efficiently against viral and bacterial infections, and promote the clearance of external pollutants [11].

The video images showed that the greater the volume of blood expelled, the more plumes were produced and the greater their length and apparent viscosity. The video images also showed that it was the formation of these plumes that 


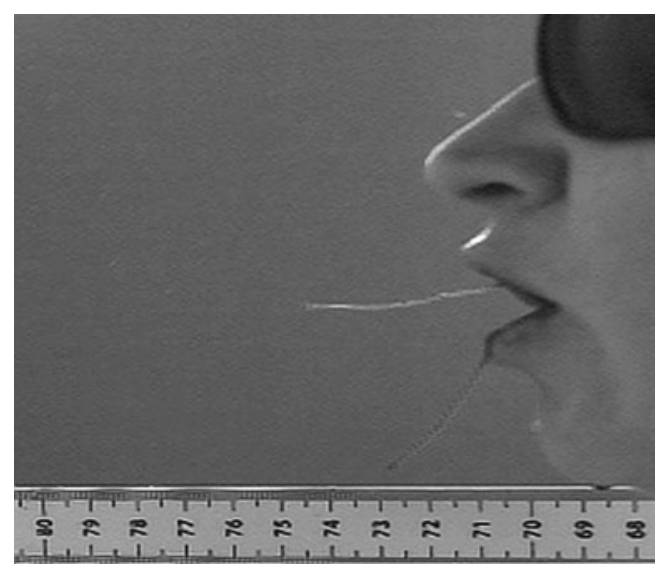

Fig. 1 Plumes attached to front teeth and bottom lip from $3 \mathrm{~mL}$ of coughed blood caused beaded bloodstains to be seen on the target surface (Fig. 2). Once plumes detached from the front teeth they began to fragment creating small droplets which resulted in 1-2-mm-sized circular stains. If a plume impacted the target surface before this process was completed, blood droplets linked by a salivary strand created beaded stains.

The formation of these beaded stains was observed mainly on the smooth non-porous cardboard target (Fig. 2), and they were comparable in appearance to the photographs of expirated stains published and described in the literature $[3,7]$ as "strings of mucus linking small blood spots".

The distance of the target was also an important factor in the formation of beaded stains. No beaded stains were seen on the vertical cardboard targets when the participant was greater than $300 \mathrm{~mm}$ away. This finding suggests that the beaded stains are only a feature of expirated bloodstain patterns if the person who produced the expirated blood is relatively close.

Observation of blood droplets impacting the target surface throughout the coughing experiments showed that air
Fig. 2 A series of images showing the formation of beaded stains. Four millilitres of blood was coughed from a standing position onto a cardboard surface from $300 \mathrm{~mm}$. The five images (a-e) are a series of still shots taken from the video demonstrating the process of beaded stains being produced from expirated plumes. The sixth image (f) is a close up of a beaded stain, (arrowed) and bubble rings (circled)

\section{a}
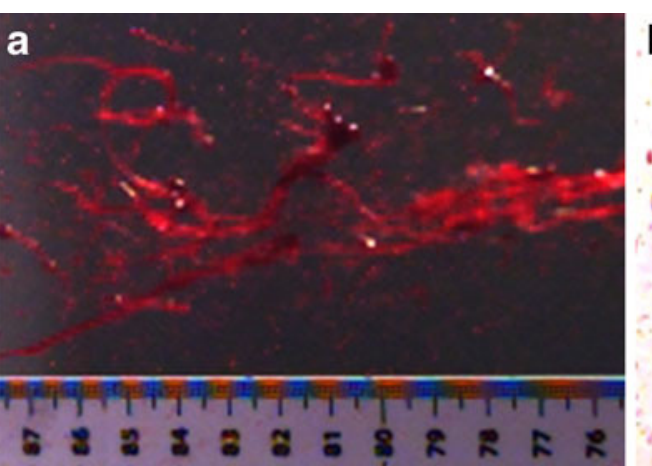

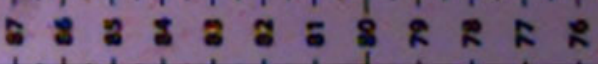
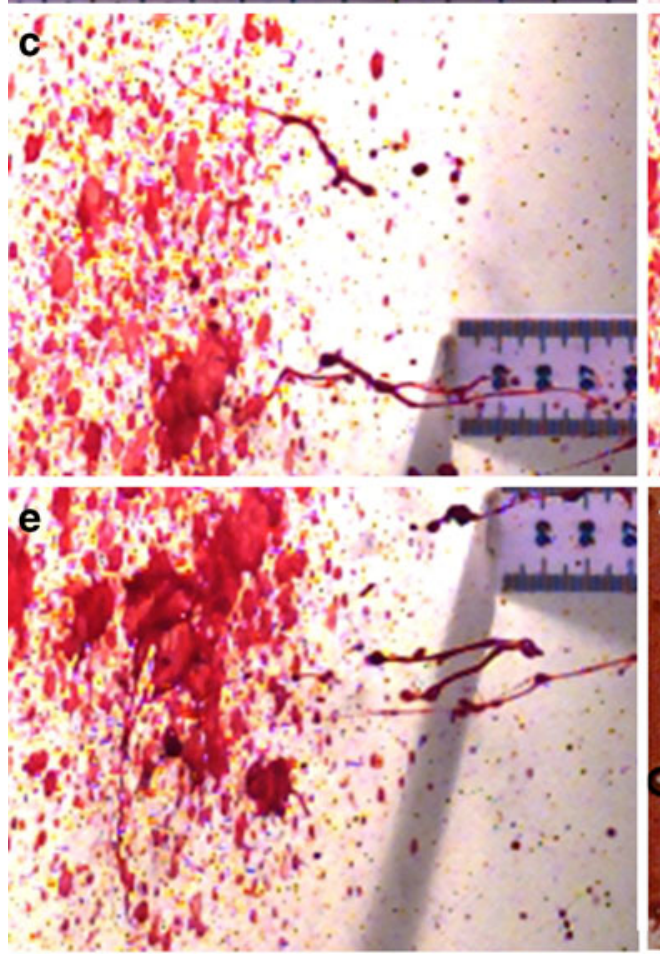



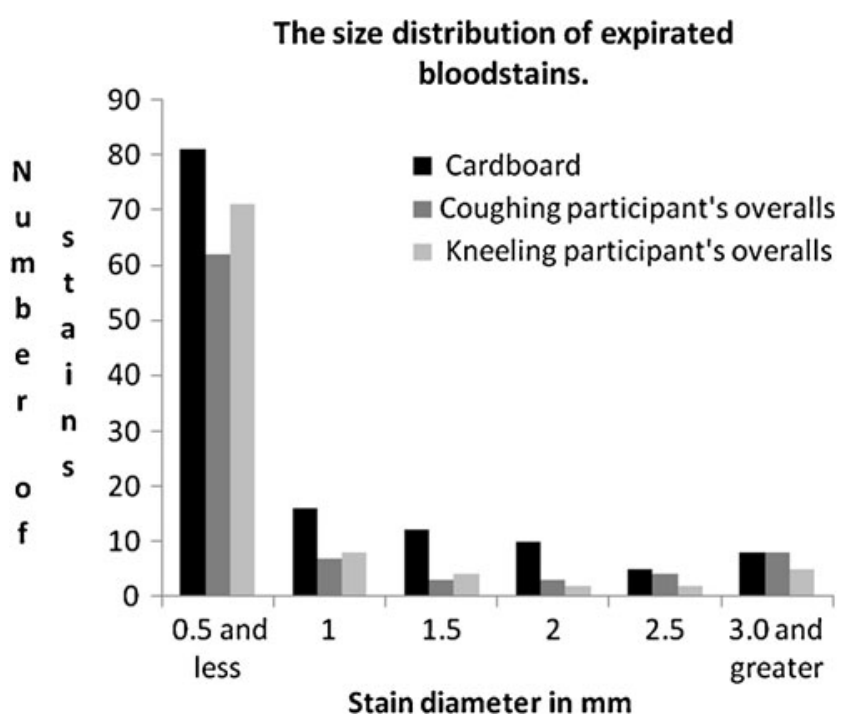

Fig. 3 The size distribution of expirated bloodstains on cardboard and Tyvex fabric for a source to target distance of $300 \mathrm{~mm}$

bubbles that were contained within a droplet frequently divided into smaller air bubbles before bursting and leaving a clear area in the centre of the droplet, creating the bubblering characteristic that has been described in the literature $[3$, 7] and can be seen in Fig. 2f. This bubble-ring characteristic was more frequently observed on the non-absorbent cardboard and was only occasionally seen on the absorbent fabric in the coughing experiments. It appeared that distance was not a variable in the formation of this feature.

Pale-coloured bloodstains indicative of dilution by saliva were also produced in the coughing experiments and were clearly visible on both the fabric and cardboard. However, this was quite variable with some blood stains a uniform dark red in colour suggesting little or no dilution. This finding suggests that the blood was not in the participant's mouth long enough to mix uniformly with saliva as could be the case if a person was bleeding into the airways and coughing up blood.

Coughed bloodstains on both target surfaces were generally $0.5 \mathrm{~mm}$ or less in diameter (Fig. 3 and 4). This is consistent with case studies [2-4] and previous research [7, 8]. Larger bloodstains, $2 \mathrm{~mm}$ or greater in diameter, were also present but only in low numbers.

Expiration patterns produced by coughing extended up to $137 \mathrm{~cm}$ by $175 \mathrm{~cm}$ in area, for a source to target distance of $1 \mathrm{~m}$. During the coughing experiments it was noted that blood drops were projected horizontally up to $175 \mathrm{~cm}$ from the mouth of the prone participant.

\section{Spitting experiments}

All experiments involving the spitting of blood showed that the tongue and lips played a major role in the ejection of blood. The video images showed that tiny mist-like blood droplets were produced in one of two ways; either the droplets were formed by blood being projected off the lips and/or tongue (Fig. 5b), or blood droplets were formed from a forward-moving sheet of fluid (Fig. 5a) which fragmented and eventually dispersed once the air pressure behind the fluid (from the force of spitting) overcame the cohesive forces within the blood.

The volume of blood present in the participant's mouth determined whether the tiny mist-like droplets were caused by being projected off the lips or by the forward-moving sheet of fluid. For larger volumes (e.g. $3-4 \mathrm{~mL}$ ), the tiny mist-like droplets appeared to be mainly produced from the fluid sheet. In smaller volumes (e.g. 1-2 mL), the fluid sheet was not as prominent and it appeared that the mistlike droplets were caused by air rushing over the surface of the fluid in the mouth and lips. The spitting experiments produced bloodstains of a similar range of sizes as those for coughing, with most stains being less than $0.5 \mathrm{~mm}$ in diameter. Expiration patterns produced by spitting extended up to $100 \mathrm{~cm}$ by $110 \mathrm{~cm}$ in area for a source to target distance of $300 \mathrm{~mm}$.

\section{Breathing experiments}

The breathing experiments indicated that the only distinctive physical characteristic observed in the resulting expirated

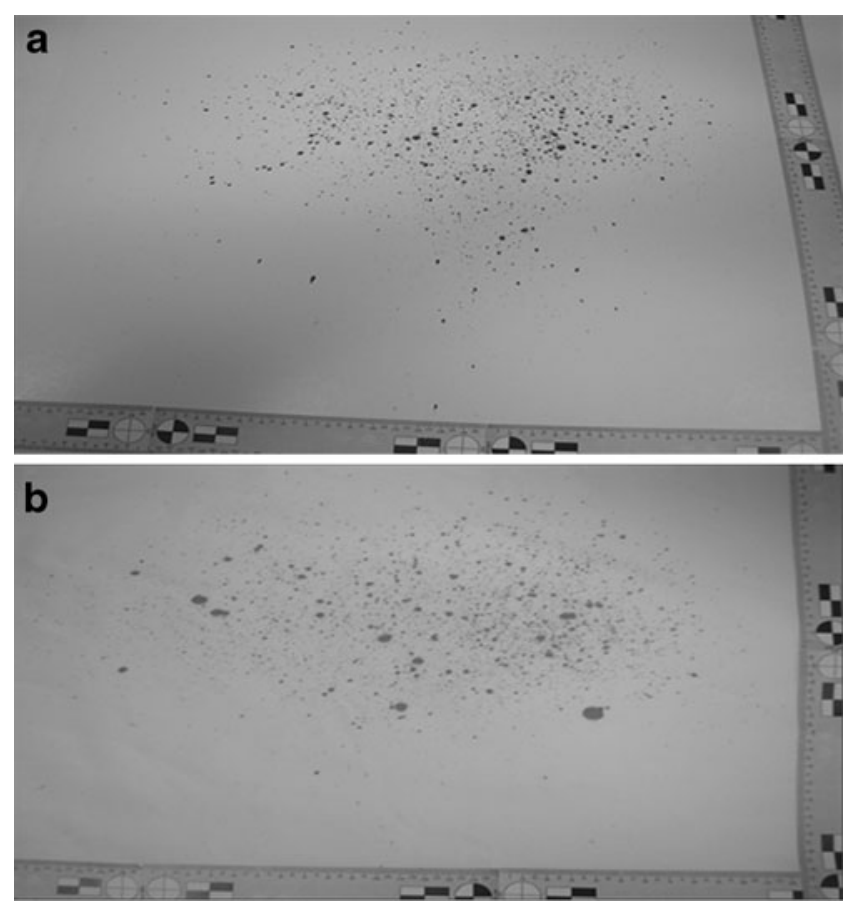

Fig. 4 Typical expirated bloodstain patterns generated on cardboard (a) and cotton fabric (b). Five millilitres of blood was coughed from a standing position onto cardboard and cotton fabric surfaces from $300 \mathrm{~mm}$ 
Fig. 5 Formation of tiny mistlike stains. a Four millilitres of blood was expelled while the participant was prone. The formation of the fluid sheet is evident. b One millilitre of blood expelled while the participant was prone. The presence of the tiny mist-like droplets are indicated by arrows in $\mathbf{a}$ and $\mathbf{b}$
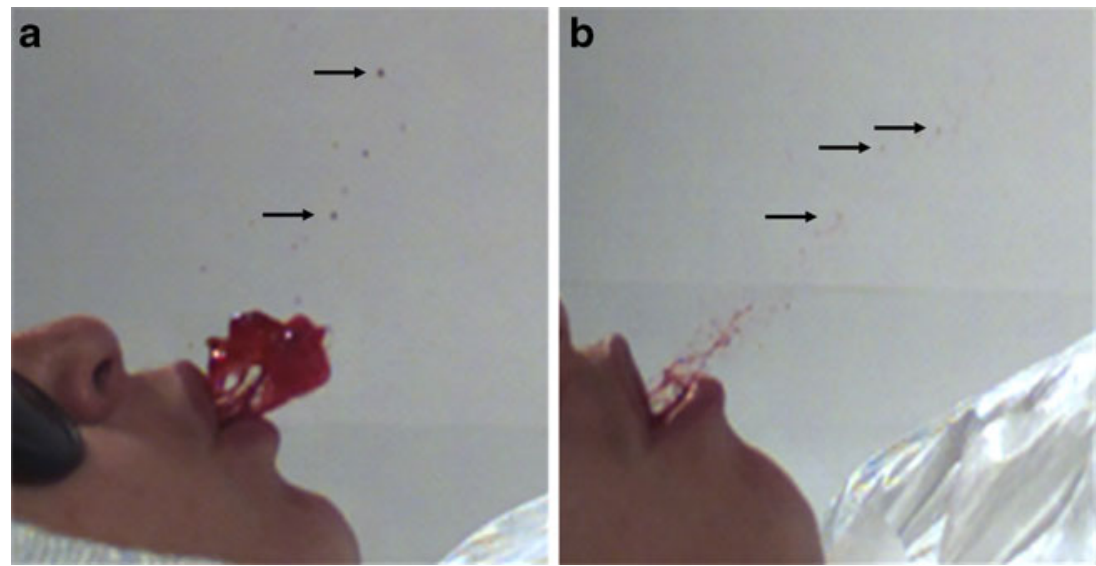

blood patterns was the presence of bubble rings; no beading or dilution was seen (Fig. 6). However, bubble rings were only observed in larger bloodstains ( $>3 \mathrm{~mm}$ in diameter). The breathing experiments produced bloodstains of a similar range of sizes as those for coughing and spitting, with most stains being less than $0.5 \mathrm{~mm}$ in diameter. Expiration patterns produced by breathing extended up to $60 \mathrm{~cm}$ by $80 \mathrm{~cm}$ in area for a source to target distances of $300 \mathrm{~mm}$.

\section{Discussion}

Forensic investigators study the shapes, sizes and locations of bloodstains in order to interpret the events that give rise to their origin. Medical findings about injuries can be used to complement these interpretations especially for suspected expirated blood. MacDonell [12] claimed that the recognition

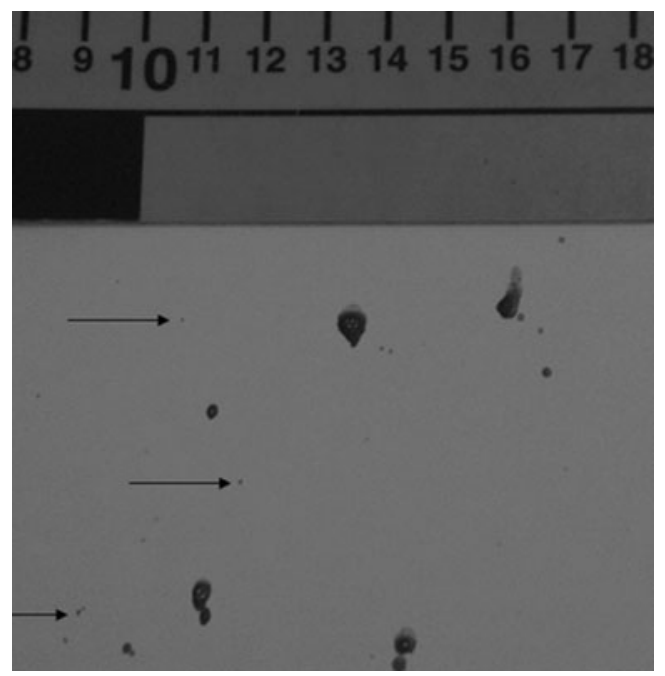

Fig. 6 Breath-expirated bloodstain pattern. One millilitre of blood was dripped down the nose and top lip while the participant breathed normally with a slightly opened mouth, spraying the blood onto the cardboard target $150 \mathrm{~mm}$ away. The presence of tiny mist-like droplets are indicated by arrows of expirated bloodstain patterns is not difficult and that if the victim has no blood in their nasal or oral orifices then it is impossible for blood to have been expirated from either source. However, cases where the victim is not found, where body decomposition is beyond a medical examination or where only partial patterns of a few bloodstains on absorbent surfaces such as clothing items are available, it is important for analysts to know the extent to which conclusions can be drawn from the bloodstain pattern alone. To this end, finding characteristic features of such patterns and their genesis during pattern formation is essential to reliable conclusions.

The high-speed digital video analysis confirms that the physical characteristics described in the literature, such as beaded stains and bubble rings [3, 7], are produced in the expiration process and are therefore useful characteristics for the identification of expirated blood from the mouth. Some caution is required with respect to beaded stains and their interpretation. These stains were not always present and generally only occurred when the target surface was within $300 \mathrm{~mm}$ of the source. They were mainly observed on smooth non-porous surfaces. The fact that beaded stains were rarely observed on the fabric surfaces is probably the result of the absorbance of blood into the fabric breaking the thin strands of liquid linking the larger stains. Furthermore, the formation of strands is thermodynamically unfavourable which means they will tend to break up to favour the more preferred spherical droplet shape as was observed in video sequences in this study. However, a target surface distance of $300 \mathrm{~mm}$ was close enough that some of the blood strands did not have time to break up and so impacted the surface with the strand still intact, resulting in a beaded stain. These findings indicate that although beaded bloodstains can be used to help identify a mouthexpirated bloodstain pattern, they will only be seen if expiration of the blood is within close proximity to the target and generally on non-porous smooth surfaces. This study also showed that beaded stains and diluted bloodstains are unlikely to be present, if at all, in expirated patterns produced by gentle breathing of blood. 
Expirated patterns produced in this study were dominated by very small stains, less than $0.5 \mathrm{~mm}$ in diameter. These tended to be from blood droplets that were ejected at high velocity at an early stage in the expiration process. Expiration causes blood droplets to project outwards in a radial fashion. This means that the resulting patterns will vary in size depending on the distance between the source and the target surface. This study shows that patterns up to $137 \mathrm{~cm}$ across are possible for a source to target distance of $1 \mathrm{~m}$, when coughing is the predominant mechanism. The results presented here are based on experiments using only one participant as the source of expirated blood; therefore, some caution is advised in generalising these results. Further work is required to validate and extend these preliminary observations.

Acknowledgments AED was supported by a postgraduate scholarship from The Institute of Environmental Science and Research Ltd. and a University of Otago postgraduate publishing bursary.

\section{References}

1. Scientific Working Group on Bloodstain Pattern Analysis (2009) Scientific working group on bloodstain pattern analysis: recommended terminology. Forensic Science Communications 11
2. Gunn A (2006) Essential Forensic Biology ed. John Wiley and Sons Ltd, West Sussex

3. James S, Kish P, Sutton T (2006) Principles of Bloodstain Pattern Analysis: Theory and Practice ed. CRC Press, New York

4. Wade K, Holmes J, Forde A, Griffiths P (2001) Death and the maiden. Sci Justice 41:64-66

5. Brown M, Wilson P (1992) How Science had to catch up. Alexander Mcleod-Lindsay waits 26 years for justice: Justice and Nightmares Successes and failures of Forensic Science in Australia and New Zealand. New South Wales University Press Ltd., UK, pp 40-60 (Ed)

6. Bevel T, Gardener RM (2008) Bloodstain Pattern Analysis with an introduction to Crime Scene reconstruction, 3rd edn. CRC Press, NewYork

7. Emes A (2001) Expirated blood — a review. Can Soc Forensic Sci J 34:197-203

8. Emes A, Price C (2004) Blood pattern analysis. Crime scene to court: the essentials of forensic Science. In: White PC (ed) 115141. Royal Society of Chemistry, Cambridge

9. Donaldson A, Taylor M, Cordiner S and Lamont I (2010) Using oral microbial DNA analysis to identify expirated bloodspatter. Int J Legal Med (in press)

10. Laber TL, Epstein BP and Taylor MC (2008) High speed digital video analysis of bloodstain pattern formation from common bloodletting mechanisms. IABPA News 2008 (June), pp 4-12

11. Wu AM, Csako G, Herp A (1994) Structure, biosynthesis, and function of salivary mucins. Mol Cell Biochem 137: 39-55

12. MacDonell HL (2005) Bloodstain patterns, 2nd edn. Golos Printing, New York 